\title{
Translation and psychometric testing of Cancer Survivors' Unmet Needs, Chinese version
}

\author{
Weijie XING ${ }^{1,2}$, Winnie Kwok Wei $\mathrm{SO}^{2}$, Kai Chow $\mathrm{CHOI}^{2}$, Cho Lee WONG ${ }^{2}$, Man \\ TONG $^{3}$, Yin Ping CHOY ${ }^{4}$, Alex MOLASSIOTIS ${ }^{5}$, Patsy YATES ${ }^{6}$, Raymond Javan \\ $\mathrm{CHAN}^{7}$
}

\begin{abstract}
Affiliations
${ }^{1}$ School of Nursing, Fudan University, China

2 The Nethersole School of Nursing, Faculty of Medicine, The Chinese University of Hong Kong, Hong Kong, China

${ }^{3}$ Department of Clinical Oncology, Tuen Mun Hopsital, Hong Kong, China

${ }^{4}$ Department of Oncology, Princess Margaret Hospital, Hong Kong, China

${ }^{5}$ School of Nursing, Hong Kong Polytechnic University, Hong Kong, China

${ }^{6}$ School of Nursing, Queensland University of Technology, Australia

${ }^{7}$ Princess Alexandra Hospital, Metro South Hospital and Health Service and School of Nursing, Queensland University of Technology, Australia
\end{abstract}

\section{Running title}

Psychometric testing of CaSUN (Chi) 


\title{
Corresponding author
}

\author{
Winnie K.W.SO \\ Address: The Nethersole School of Nursing, Faculty of Medicine, The Chinese \\ University of Hong Kong, 7/F, Esther Lee Building, The Chinese University of Hong \\ Kong, Shatin, N.T., Hong Kong. \\ Tel: (852) 39431072. \\ Fax: (852) 26036041. \\ Email address: winnieso@cuhk.edu.hk.
}

\section{Acknowledgements}

Nil

\section{Funding}

The authors received no funding.

\section{Conflict of Interest}

The authors declare no conflicts of interest. 


\begin{abstract}
Aim: The aim of the study was to translate the Cancer Survivors' Unmet Needs (CaSUN) scale into Chinese, and then test its psychometric properties, for cancer survivors in Hong Kong.

Methods: The original questionnaire was translated from English into traditional Chinese (CaSUN-Chi), following standardized procedures. An expert panel was invited to assess the items' content validity, and pilot test on 15 patients to evaluate its readability. The sample for psychometric evaluation was drawn from a large multi-national study assessing unmet needs of cancer survivors, with a convenience sample of 300 was recruited. Cronbach's alpha coefficient was used to assess the internal consistency of the scale, and confirmatory factor analysis to evaluate its construct validity.
\end{abstract}

Results: The CaSUN-Chi had good readability and high content validity (S-CVI 0.98). Cronbach's alpha for the entire scale was 0.93 and 0.71 to 0.91 for the five sub-scales. Confirmatory factor analysis indicated that the five-factor structure of the CaSUN-Chi was good fit to the data $(\mathrm{CFI}=0.99, \mathrm{AGFI}=0.98, \mathrm{RMSEA}=0.054, \mathrm{SRMR}=0.071)$.

Conclusion: The CaSUN-Chi showed desirable psychometric properties for assessing unmet needs of cancer survivors in Hong Kong. Using the newly translated scale to identify individual supportive care unmet needs can bridge the gap between patients' experiences and expectations, and improve healthcare provision and resource allocation. 
Keywords: cancer survivors, Chinese, psychometric validation, supportive care needs, unmet needs 


\section{Background}

According to an estimate by the International Association of Cancer Registries, there were more than 14 million new cancer cases and eight million deaths in $2012 .{ }^{1}$ With continuing advances in cancer detection and treatment, an increasing number of people are surviving the disease. ${ }^{2}$ In 2012, more than 32 million people globally were living with cancer. ${ }^{3}$ In Hong Kong, the number of new cancer cases has been rising by $2.4 \%$ a year, and reached a historic high of 30,318 in $2015 .{ }^{4}$ Meanwhile, the cancer survival rate has doubled in the last 40 years and nearly half of cancer patients are expected to survive for at least 10 years. ${ }^{5}$

Survivors experience numerous physical and psychosocial problems as a result of the cancer diagnosis and treatment. ${ }^{6}$ Supportive care needs refers to the various forms of care required by patients with cancer and their families, from first diagnosis to cure, or death and bereavement. ${ }^{7}$ Many survivors report having unmet needs, especially related to the daily living activities, information, and physical and psychological symptoms. ${ }^{7-9}$ Patientperceived unmet supportive care needs are associated with a decreased quality of life..$^{9-12}$ Understanding these unmet needs not only bridges the gap between patients' experiences and expectations, but also improves healthcare provision and resource allocation.

The Supportive Care Needs Survey (SCNS), Survivors Unmet Needs Survey (SUNS) and Cancer Survivor's Supportive Unmet Needs (CaSUN) are the most commonly used scales for assessing cancer survivors' supportive care needs. SCNS is originally a 60 -item scale ${ }^{13}$ and was modified to a short form of 34 items (SCNS-SF 34) by Boyes et al, with 
a good reliability and validity. ${ }^{14}$ Although SCNS-SF 34 has been translated into traditional Chinese, it is specifically validated for breast 15 and colorectal cancer survivors ${ }^{16}$. In addition, SCNS does not specifically focus on the survivorship phase, with a majority of items assessing care needs during acute cancer treatment. SUNS is a 89-item instrument, ${ }^{17}$ modified to a short-form by Campbell et al ${ }^{18}$ and Taylor et al ${ }^{19}$. It has good psychometric properties and covers five sub-scales (emotional health needs, access and continuity of care, relationships, financial concerns and information needs). This instrument primarily focuses on the unmet needs of early-stage survivors one to five years after diagnosis. In addition, there are eight items in the financial concerns sub-scale, which may not be applicable to other socio-cultural backgrounds. CaSUN is a 35 -item questionnaire, developed to evaluate the supportive care needs of cancer survivors. It includes five domains (existential survivorship, comprehensive cancer care, information, quality of life and relations) and is more appropriate for addressing the demands of the survivorship in various disease trajectories. ${ }^{20} \mathrm{CaSUN}$ has been translated into Spanish and Dutch and modified and shown to be a valid and reliable assessment tool. ${ }^{21,22}$ It has also been modified and validated in Taiwanese Chinese by Fang et al, ${ }^{23}$ but this version was only validated using a group of breast cancer survivors. Translation and validation of the instrument in a general Chinese cancer survivor population has yet to be done. Besides, instead of using the conceptual framework of the original instrument, for validation, the authors conducted exploratory factor analysis and re-categorized the scale into four domains (information, physical/psychological, medical care and communication). Translation and validation of the Chinese version of this instrument, 
using the original construct and a sample of cancer survivors of various cancer types, is therefore required.

\begin{abstract}
Above all, unmet needs assessment will help healthcare professionals to target survivors' individual needs, and lead to optimal care delivery. There is a lack of appropriate instruments to evaluate the multi-dimensional supportive care needs of Chinese cancer survivors in Hong Kong. The aim of this study was to translate the CaSUN scale into Chinese and then test its psychometric properties among cancer survivors in Hong Kong.
\end{abstract}

\title{
Methods
}

\section{Design}

The study included two phases. First, the CaSUN was translated from English into Chinese following standard procedure, and then tested for its content validity. Second, certain crucial psychometric properties of CaSUN-Chi, including internal consistency and construct validity, were assessed.

\section{Phase 1: Translation and content validation}

The translation of CaSUN was guided by the World Health Organization guidelines on the process of translation and adaptation of instruments. ${ }^{24}$ First, forward translation was carried out by a bilingual nurse educator who independently translated the original scale from English into Chinese. Another independent bilingual member of the research staff, 
who had no knowledge of the questionnaire, translated the first Chinese version back into English. This back-translated version was compared by the second author (WKW) with the original version. Discrepancies between the two versions were identified and their consistency of meaning and cultural equivalence discussed.

\begin{abstract}
A panel of four expert professionals (two nursing faculty members, one nurse consultant and one social worker) was then invited to evaluate the items' relevance to the scale and local clinical context. Each item was rated on a four-point scale $(1=$ not relevant, $2=$ somewhat relevant, $3=$ relevant, $4=$ highly relevant). As suggested by Polit and Beck, ${ }^{25}$ the content validity index (CVI) was computed on two levels: item-level CVI (I-CVI) and scale-CVI (S-CVI). The item-level CVI was determined by the proportion of experts who rated it 3 or 4 , and the scale-CVI by the proportion of items with full agreement on their relevance by the panel. An I-CVI score of 0.78 and S-CVI of 0.90 were the minimum acceptable indices. $^{25}$
\end{abstract}

Finally, a pilot test with a convenience sample of 15 cancer patients was conducted to evaluate the readability of CaSUN-Chi. Each patient was asked to rate the instructions, response format and items of the scale for clarity, making a simple dichotomous choice (clear or unclear). Participants rating something unclear were further interviewed to provide suggestions on how to rewrite the statements more clearly. ${ }^{26}$

\title{
Phase 2: Psychometric testing of CaSUN-Chi
}




\title{
Participants and study setting
}

The sample for psychometric evaluation was drawn from a large multi-national study assessing unmet needs among cancer survivors. ${ }^{27}$ A total of 300 patients were recruited from the outpatient departments of clinical oncology at two Hong Kong public hospitals, using a convenience sampling method. Inclusion criteria were cancer patients who (1) were 18 or above, (2) were diagnosed with cancer, (3) had completed all first-line treatment and (4) were able to read Chinese or communicate in Cantonese. Patients with cognitive impairment or language difficulties were excluded.

\begin{abstract}
Based on the power analysis for the statistical test, which was the confirmatory factor analysis involved in this study, the minimum sample size recommended is at least five participants per parameter estimate, so that the sample size of 300 was able to achieve accuracy in estimating the model parameters. ${ }^{28}$
\end{abstract}

\section{Measurements}

A self-report questionnaire was developed to collect demographics (gender, age) and disease-related characteristics (time since diagnosis, specific sites of the disease and treatment received) from participants.

The Chinese version (CaSUN-Chi) was used to assess the unmet needs of cancer survivors. CaSUN-Chi consists of 35 items, 28 of which, based on the validity study by the original author, ${ }^{20}$ can be categorized into five domains: existential survivorship (14 
items), comprehensive cancer care (6 items), information (3 items), quality of life (2 items) and relationships (3 items). The remaining seven items are not grouped into a domain, and can be used to measure clinically useful information. Participants were asked whether the needs described by the items were met, unmet or not applicable. Each item can be scored in terms of met, unmet and total needs. If an unmet need is reported, its strength is marked as 'weak', 'moderate' or 'strong'. The domains are scored by summing all the items it contains, with the average number of needs in a domain reported. The total scores consist of the sum of all need items, with higher scores indicating greater needs (range $0-35$ ).

\section{Ethical considerations}

Ethical approval of the study was obtained from the institutional review board of the study institutions. The study researchers sought permission for access to the medical records of the patients. Eligible patients were approached by research staff at their regular medical consultations. If they were interested in participating, the research team contacted them to obtain their informed consent.

\section{Data collection}

Patients were invited to complete the demographic questionnaire and CaSUN-Chi independently, while they were waiting to be seen by the doctor, and then returned the questionnaires to the researcher as soon as they were completed. A quality check was carried out immediately to assess the validity of the questionnaire. 


\section{Data analysis}

Patients' demographic and disease-related characteristics were summarized and reported by frequency, percentage, mean and SD, as appropriate. The scale's internal consistency was assessed by means of Cronbach' alpha, and a coefficient $>0.7$ was considered to indicate satisfactory internal consistency. ${ }^{25}$ The five-factor structure of the CaSUN-Chi was examined by confirmatory factor analysis (CFA) using LISREL 8.8 (Scientific Software International, Inc.). The parameters of the CFA model were estimated by the robust diagonally weighted least squares method, which allows violation of the multivariate normality assumption of the data. Since chi-square tests are sensitive to sample size and violation of the multivariate normality assumption, ${ }^{29}$ several goodnessof-fit indices were used to assess the overall fit of the model. Guided by Schreiber., ${ }^{29}$ the following fit indices were chosen: the Satorra-Bentler scaled chi-square statistic to degree of freedom ratio $\left(\chi^{2 / d f}\right)$, root mean square error of approximation (RMSEA), standardized root mean square residual adjusted (SRMR), comparative fit index (CFI) and adjusted goodness-of-fit index (AGFI). Acceptable model fit was determined with an SRMR value of $\leq 0.08$, RMSEA value of $\leq 0.05$ and values $\geq 0.95$ for CFI and AGFI. ${ }^{29}$ Except CFA, all statistical analyses were performed using IBM SPSS 24 (IBM Corp., Armonk, NY).

\section{Results}

\section{Phase I Translation and content validation}


The researchers identified any discrepancies in items between the translated Chinese, back-translated English and original versions of CaSUN, and then clarified them by discussion. Minor wording was modified in two items, including from 'adapt' to 'adjust' in item 12, and from 'talk' to 'communicate' in item 24 . Otherwise, there were no additional amendments to the scale.

The content validity of CaSUN-Chi was confirmed by the panel members. They found CaSUN-Chi to be clearly written, easy to understand and highly relevant to the topic of unmet supportive care needs in the local practice context. Nearly all items were rated 1 for I-CVI, except for three $(16,17$, and 18$)$ that were rated 0.75 . The S-CVI was accorded scores of 0.98 . Three items that were not scored 1 by all experts were: 'Due to my cancer, I need help getting life and/or travel insurance' (item 16), 'Due to my cancer, I need help accessing legal services' (items 17), 'I need more accessible hospital parking' (item 18). One expert rated these three items as 'somewhat relevant', explaining that the needs mentioned in these items were not necessarily required by cancer survivors in Hong Kong. However, after discussion among panel members, researchers and representative patients, all three were retained in the scale, by consensus.

\begin{abstract}
As for cognitive debriefing, all 15 patients agreed that the Chinese version of CaSUN was clear and easily understood, and no offensive words were included. It took about 15 minutes to complete all the items.
\end{abstract}

\title{
Phase II Psychometric properties
}




\section{Participant characteristics}

The mean age of participants was $55.6 \pm 12.3$, with a range from 21 to 89 years. The majority $(211,70.3 \%)$ were female. Participants were diagnosed with various types of cancer - breast cancer (30.3\%), gynecological (24.3\%), head and neck (21.3\%), gastric or colorectal cancer $(10.7 \%)$. The average time since diagnosis was $6.3 \pm 4.6$ years. The $\underline{\text { median time since diagnosis was five years, with an interquartile range between three and }}$ eight years. The range of the time since diagnosis was $1-22$ years.

\section{Internal consistency}

Cronbach's alpha for the entire CaSUN-Chi scale was 0.93. None of the items would have a significant impact on the scale alpha if it was removed. Cronbach's alpha coefficients of the sub-scales of existential survivorship, comprehensive cancer care, information, quality of life and relationship domains were $0.91,0.82,0.81,0.78$ and 0.71 respectively.

\section{Construct validity}

The construct validity of CaSUN-Chi was evaluated by confirmatory factor analysis. Figure 1 shows the results of the CFA. Standardized factor loadings ranged from 0.36 to 0.89 , and error variances from 0.20 to 0.87 . The model showed a good fit to the data with $\chi 2 / \mathrm{df}=1.86, \quad \mathrm{CFI}=0.99, \quad$ AGFI $=0.98, \quad \mathrm{RMSEA}=0.054 \quad(90 \% \quad$ CI $\quad 0.047-0.060)$, $\mathrm{SRMR}=0.071$. 


\section{Discussion}

The present study translated CaSUN into Chinese and determined its psychometric properties, and the results of CVI, Cronbach's alpha and CFA demonstrated that it had desirable reliability and validity.

The CaSUN was translated into Chinese using a rigorous procedure recommended by $\mathrm{WHO},{ }^{24}$ involving forward-backward translation, expert panel content validation and cognitive debriefing of a representative sample. The results showed a scale-CVI of 0.98 , much higher than the excellence criterion of $0.90,{ }^{25}$ indicating that CaSUN-Chi was suitable for the construct, related population and socio-cultural background of the study setting.

As for reliability, with Cronbach's alphas of 0.93 for the scale and 0.70 or above for all five sub-scales, the CaSUN-Chi displays high internal consistency, comparable with the original version by Hodgkinson et al, ${ }^{20}$ the Spanish evaluated by Martinez Tyson et al. ${ }^{21}$ and the extended Dutch version modified by Keeman et al. ${ }^{22}$ A Cronbach's alpha value of 0.7 is recognized as adequate, and one of 0.8 is deemed highly satisfactory. ${ }^{25}$ The high internal consistency of CaSUN in different language contexts provided evidence for its strong reliability.

Following current recommendations, acceptable model fit was determined by an SRMR value of $\leq 0.08$, an RMSEA value of $\leq 0.05$ and values greater than 0.95 for CFI and 
AGFI. ${ }^{29}$ The CFA result indicated that the original five-factor structure was a reasonably good fit to the data of Hong Kong cancer survivors. Although the five-factor model has been confirmed, item 18 (accessible hospital parking) had the lowest factor loading (0.36) and the largest residual error (0.87). In Hong Kong, the public transport system is well developed and driving is not necessary when going to hospital. Only eight patients reported unmet needs in this item. In the Spanish version ${ }^{21}$ and another Chinese version modified for Taiwan breast cancer survivors ${ }^{23}$, this item was not included. So, whether accessible hospital parking is necessary to cancer survivors appears to be societydependent and may be reconsidered in any future study.

\section{Study strengths and limitations}

The study used a standard translation process and systematic validation methods to provide evidence for the CaSUN-Chi as a useful measure of Chinese cancer survivors' unmet needs. However, some limitations should be noted. First, due to the cross-sectional design of the study, test-retest reliability and criteria validity were not examined. Second, although the CFA supported the original model of the scale, there still existed some items with lower factor loadings and larger residual errors. Future research should examine the test-retest reliability and convergent and criteria-related validity of CaSUN-Chi.

\section{Conclusions and implications for nursing practice}

The CaSUN-Chi showed desirable psychometric properties for assessing the unmet needs of cancer survivors in Hong Kong. Using the instrument to identify individual supportive 
care unmet needs can bridge the gap between patients' experience and expectations, and improve healthcare provision and resource allocation.

\section{Acknowledgements}

Nil 


\section{References}

1. Torre LA, Bray F, Siegel RL, Ferlay J, Lortet-Tieulent J, Jemal A. Global cancer statistics, 2012. CA Cancer J Clin 2015; 65: 87-108.

2. Miller KD, Siegel RL, Lin CC, et al. Cancer treatment and survivorship statistics, 2016. CA Cancer J Clin 2016; 66: 271-89.

3. Bray F, Ren JS, Masuyer E, Ferlay J. Global estimates of cancer prevalence for 27 sites in the adult population in 2008. Int J Cancer 2013; 132: 1133-45.

4. Hong Kong Cancer Registry. Overview of Hong Kong Cancer Statistics of 2015. 2017; http://www3.ha.org.hk/cancereg/. Accessed Mar 14, 2018.

5. Cancer Research UK. Cancer survival statistics. http://www.cancerresearchuk.org/health-professional/cancer-statistics/survival. Accessed March 14, 2018.

6. Moser EC, Meunier F. Cancer survivorship: A positive side-effect of more successful cancer treatment. EJC Suppl 2014; 12: 1-4.

7. Harrison JD, Young JM, Price MA, Butow PN, Solomon MJ. What are the unmet supportive care needs of people with cancer? A systematic review. Support Care Cancer 2009; 17: 1117-28.

8. Wiley G, Piper A, Phyllis Butow AM, et al. Developing Written Information for Cancer Survivors from Culturally and Linguistically Diverse Backgrounds: Lessons Learnt. Asia Pac J Oncol Nurs 2018; 5: 121-126.

9. So WK, Chan CW, Choi KC, Wan RW, Mak SS, Chair SY. Perceived unmet needs and health-related quality of life of Chinese cancer survivors at 1 year after treatment. Cancer Nurs 2013; 36: E23-E32. 
10. So WK, Choi KC, Chen JM, et al. Quality of life in head and neck cancer survivors at 1 year after treatment: the mediating role of unmet supportive care needs. Support Care Cancer 2014; 22: 2917-2926.

811. Scotte F. The importance of supportive care in optimizing treatment outcomes of patients with advanced prostate cancer. Oncologist 2012; 17: 23-30.

912. Malmstrom M, Ivarsson B, Klefsgard R, Persson K, Jakobsson U, Johansson J. The effect of a nurse led telephone supportive care programme on patients' quality of life, received information and health care contacts after oesophageal cancer surgery-A six month RCT-follow-up study. Int J Nurs Stud 2016; 64: 86-95.

1013. Bonevski B, Sanson-Fisher R, Girgis A, Burton L, Cook P, Boyes A. Evaluation of an instrument to assess the needs of patients with cancer. Supportive Care Review Group. Cancer 2000; 88: 217-25.

1414. Boyes A, Girgis A, Lecathelinais C. Brief assessment of adult cancer patients' perceived needs: development and validation of the 34-item Supportive Care Needs Survey (SCNS-SF34). J Eval Clin Pract 2009; 15: 602-6.

1215. Au A, Lam WW, Kwong A, et al. Validation of the Chinese version of the shortform Supportive Care Needs Survey Questionnaire (SCNS-SF34-C). Psychooncology 2011; 20: 1292-300.

1316. Li WW, Lam WW, Shun SC, et al. Psychometric assessment of the Chinese version of the Supportive Care Needs Survey short-form (SCNS-SF34-C) among Hong Kong and Taiwanese Chinese colorectal cancer patients. PLoS One 2013; 8: e75755. 
1417. Campbell HS, Sanson-Fisher R, Turner D, Hayward L, Wang XS, Taylor-Brown J. Psychometric properties of cancer survivors' unmet needs survey. Support Care Cancer 2010; 19: 221-30.

1518. Campbell HS, Hall AE, Sanson-Fisher RW, Barker D, Turner D, Taylor-Brown J. Development and validation of the Short-Form Survivor Unmet Needs Survey (SF-SUNS). Support Care Cancer 2014; 22: 1071-9.

1619. Taylor K, Bulsara M, Monterosso L. Test-Retest Reliability of the Short-Form Survivor Unmet Needs Survey. Asia Pac J Oncol Nurs 2018; 5: 165-71.

1720. Hodgkinson K, Butow P, Hunt GE, et al. The development and evaluation of a measure to assess cancer survivors' unmet supportive care needs: the CaSUN (Cancer Survivors' Unmet Needs measure). Psychooncology 2007; 16: 796-804.

1821. Martinez Tyson D, Medina-Ramirez P, Vazquez-Otero C, Gwede CK, Babilonia MB, McMillan SC. Initial evaluation of the validity and reliability of the culturally adapted Spanish CaSUN (S-CaSUN). J Cancer Surviv 2018. Advance online publication. doi: 10.1007/s11764-018-0689-5.

1922. Keeman MC, Bolman CAW, Mesters I, Willems RA, Kanera IM, Lechner L. Psychometric properties of the Dutch extended Cancer Survivors' Unmet Needs measure (CaSUN-NL). Eur J Cancer Care (Engl) 2018; 27: e12807.

2023. Fang SY, Cheng HR, Lin CY. Validation of the modified Chinese Cancer Survivor's Unmet Needs (CaSUN-C) for women with breast cancer. Psychooncology 2018; 27: 236-42. 
2124. World Health Organization. Process of translation and adaptation of instruments. 2013; http://www.who.int/substance_abuse/research_tools/translation/en/. Accessed Apr 13, 2018.

2225. Polit DF, Beck CT. Nursing Research: Generating and Assessing Evidence for Nursing Practice (10th edition). Wolters Kluwer, 2016.

2326. Sousa VD, Rojjanasrirat W. Translation, adaptation and validation of instruments or scales for use in cross-cultural health care research: a clear and user-friendly guideline. J Eval Clin Pract 2011; 17: 268-74.

2427. Molassiotis A, Yates P, Li Q, et al. Mapping unmet supportive care needs, quality-of-life perceptions and current symptoms in cancer survivors across the Asia-Pacific region: results from the International STEP Study. Ann Oncol 2017; 28: $2552-8$.

2528. Thompson B. Exploratory and confirmatory factor analysis: Understanding concepts and applications. American Psychological Association, Washington DC, 2004.

2629. Schreiber JB. Update to core reporting practices in structural equation modeling. Res Social Adm Pharm 2017; 13: 634-43. 


\section{Figure legends}

Figure 1: Confirmatory factor analysis of CaSUN-Chi. ES = existential survivorship, $\mathrm{CCC}=$ comprehensive cancer care, Inf $=$ information, $\mathrm{QoL}=$ quality of life, Rel $=$ relationships, CaSUN-Chi $=$ Traditional Chinese version of Cancer Survivors' Unmet Need Scale 
Figure 1: Confirmatory factor analysis of CaSUN-Chi. ES $=$ existential survivorship, $\mathrm{CCC}=$ comprehensive cancer care, Inf $=$ information, $\mathrm{QoL}=$ quality of life, Rel = relationships, CaSUN-Chi $=$ Traditional Chinese version of Cancer Survivors' Unmet Need Scale

$190 \times 338 \mathrm{~mm}(300 \times 300 \mathrm{DPI})$ 